\title{
Three $p$ 's in a single patient: percutaneous valvuloplasty, percutaneous circulatory support, and percutaneous coronary intervention
}

\author{
Krzysztof Ściborski', Michał Furdal', Marek Mak², Katarzyna Wojciechowska³, Maciej Banasiak', \\ Agnieszka Wysokińska-Kordybach', Waldemar Banasiak', Artur Telichowski' \\ 1 Department of Cardiology, 4th Military Hospital, Wrocław, Poland \\ 2 Department of Cardiac Surgery, 4th Military Hospital, Wrocław, Poland \\ 3 Department of Anesthesiology and Intensive Therapy, 4th Military Hospital, Wrocław, Poland
}

\author{
Correspondence to: \\ Krzysztof Ściborski, MD, PhD, \\ Department of Cardiology, \\ 4th Military Hospital, ul. Weigla 5, \\ 50-981 Wrocław, Poland, \\ phone: + 48261660452 \\ email: k.sciborski@op.pl \\ Received: 0ctober 16, 2020. \\ Revision accepted: \\ December 16, 2020. \\ Published online: January 4, 2021. \\ Kardiol Pol. 2021; 79 (1): 93-94 \\ doi:10.33963/KP.15738 \\ Copyright by the Author(s), 2021
}

Intra-aortic balloon pump (IABP) has been used since the 1960s as mechanical circulatory support (MCS) in cardiogenic shock, and since 1990s it has also been applied for high-risk percutaneous coronary intervention (PCI). ${ }^{1}$ Apart from IABP, alternative percutaneous left ventricular (LV) assist devices (LVADs) are available, such as extracorporeal membrane oxygenation (ECMO), Impella (Abiomed, Denvers, Massachusetts, United States), or TandemHeart (CardiacAssist, LivaNova PLC, London, United Kingdom).

Of all MCS devices, Impella seems to be the optimal choice in terms of the impact on cardiac flow (IABP, up to $1 \mathrm{l} / \mathrm{min}$; Impella and TandemHeart, up to $5 \mathrm{l} / \mathrm{min}$; extracorporeal membrane oxygenation, 3-7 1/min), implementation techniques, staff involvement, and complications. ${ }^{2}$ It is a continuous-flow device that is placed in the LV across the aortic valve $(\mathrm{AV})$, using a retrograde femoral artery access. Once implanted, it pumps blood from the LV into the ascending aorta.

High-risk PCI is defined according to several clinical features, comorbidities, and anatomical characteristics, the most important being LV systolic dysfunction (ie, LV ejection fraction [LVEF] <35\%), congestive heart failure, severe aortic stenosis (AS) and mitral regurgitation, unprotected left main disease, severe 3-vessel disease, SYNTAX score exceeding 33, last remaining patent vessel, and chronic kidney disease. To avoid hemodynamic collapse and cardiac arrest, MCS should be planned before PCI.
A 74-year-old man was referred to our center from a regional cardiology department where he was hospitalized for cardiovascular decompensation. Previous coronary angiography showed critical stenosis of the coronary arteries (FIGURE $1 A$ and 1B). Other findings included severe low-flow, low-gradient $A S\left(V_{\max }, 3.65 \mathrm{~m} / \mathrm{s}\right.$; maximum/mean gradient, 53/35 mm Hg; AV area, $0.7 \mathrm{~mm}^{2}$ ), reduced LVEF (<20\%), a EuroSCORE of $16.1 \%$, and a SYNTAX score of 58. Considering the high-risk surgery after Heart Team evaluation, the patient was scheduled for PCI with LVAD support.

As we expected problems with Impella insertion and possible AV obstruction, we decided to perform balloon valvuloplasty (FIGURE 1C). After the guidewire was inserted through the $\mathrm{AV}$, the patient developed hypotension requiring vasopressor support (norepinephrine). After valvuloplasty, Impella CP was successfully placed in the LV. Next, intravascular ultrasound-guided PCI was performed with intermediate level of support (cardiac flow, 3.5 l/min) (FIGURE 1D and 1E).

During the procedure, the patient was stable and conscious. The device was removed immediately afterwards in the catheterization laboratory, according to the protocol. At 30-day follow-up, LVEF improved to 37\%. Six weeks after valvuloplasty and PCI, transcatheter aortic valve implantation was successfully performed (FIGURE 1F), resulting in further LVEF increase to $45 \%$ at 30 days.

As the Impella system is generally contraindicated in AS, few cases of its use during high-risk 

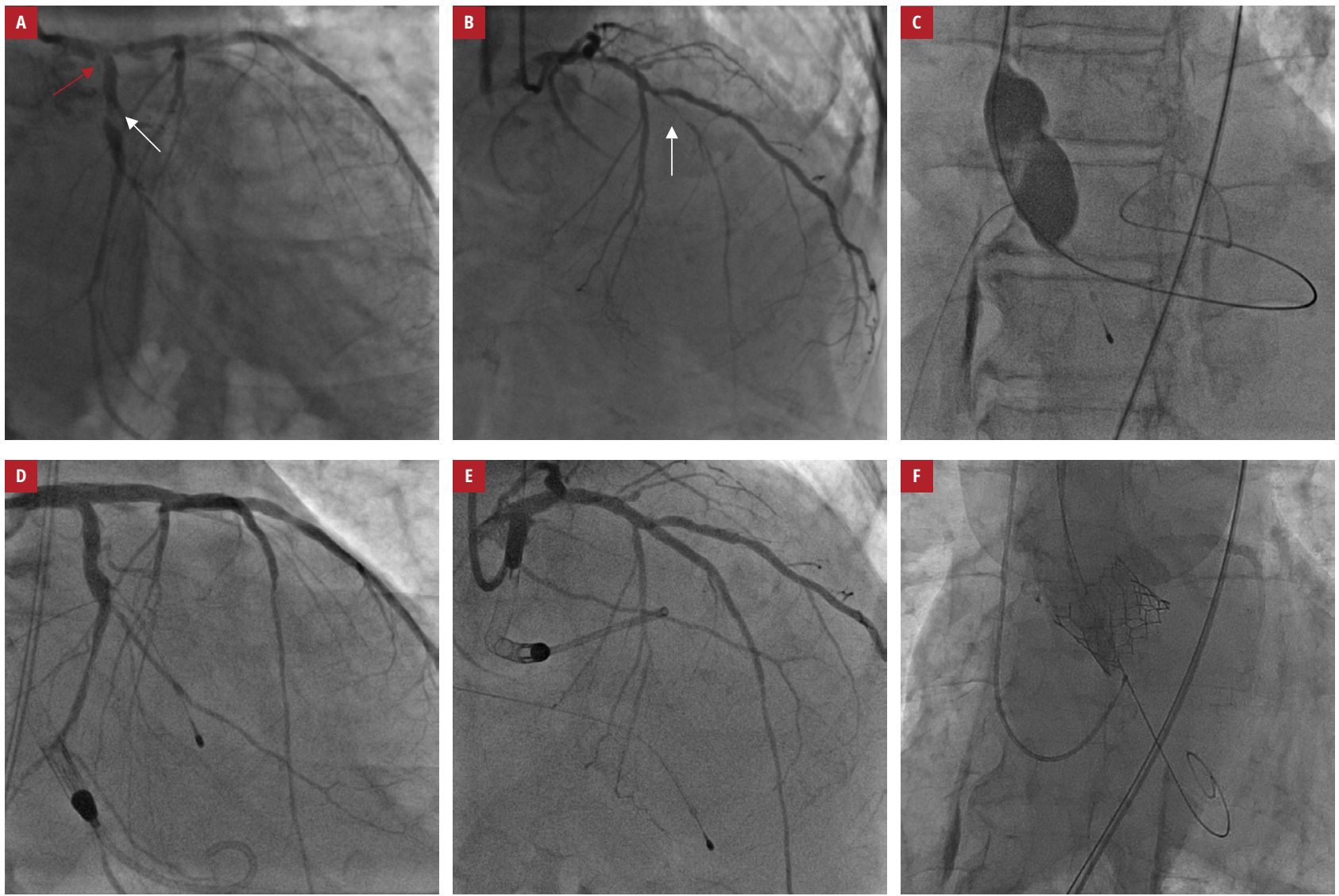

FIGURE 1 Angiography imaging: A - the left coronary artery (LCA) before percutaneous coronary intervention (PCI) showing stenosis in the distal left main coronary artery, Medina 1,1,1 (red arrow) and the circumflex artery (white arrow); B - the LCA before PCI showing critical stenosis in the left anterior descending artery (arrow); C - balloon aortic valvuloplasty; D, E - LCA after PCI, Impella in the left ventricle; F - transcatheter aortic valve implantation

PCI in these patients have been reported, and valvuloplasty was applied depending on the possibility of insertion. ${ }^{3,4}$ Another limitation of the use of Impella is peripheral arterial stenosis; however, it may also be simultaneously treated with minimally invasive methods. ${ }^{5}$ Our case shows that Impella can be successfully used in high-risk patients with AS when valvuloplasty can be performed, if necessary. This strategy appears to be feasible in this growing population of patients with AS.

\section{ARTICLE INFORMATION}

\section{CONFLICT OF INTEREST None declared.}

OPEN ACCESS This is an Open Access article distributed under the terms of the Creative Commons Attribution-NonCommercial-NoDerivatives 4.0 International License (CC BY-NC-ND 4.0), allowing third parties to download articles and share them with others, provided the original work is properly cited, not changed in any way, distributed under the same license, and used for noncommercial purposes only. For commercial use, please contact the journal office at kardiologiapolska@ptkardio.pl.

HOW TO CITE Ściborski K, Furdal M, Mak M, et al. Three $p$ 's in a single patient: percutaneous valvuloplasty, percutaneous circulatory support, and percutaneous coronary intervention. Kardiol Pol. 2021; 79: 93-94. doi:10.33963/KP.15738

\section{REFERENCES}

1 Kahn JK, Rutherford BD, McConahay DR, et al. Supported "high risk" coronary angioplasty using intraaortic balloon pump counterpulsation. J Am Coll Cardiol. 1990; 15: 1151-1155.

2 Atkinson TM, Ohman EM, O'Neill WW, et al. Interventional Scientific Council of the American College of Cardiology. A practical approach to mechanical circulatory support in patients undergoing percutaneous coronary intervention: an interventional perspective. JACC Cardiovasc Interv. 2016; 9: 871-883.

3 Martinez $C A$, Singh V, Londono JC, et al. Percutaneous retrograde left ventricular assist support for interventions in patients with aortic stenosis and left ventricular dysfunction. Catheter Cardiovasc Interv. 2012; 80: 1201-1209.

4 Singh V, Yadav PK, Eng MH, et al. Outcomes of hemodynamic support with Impella in very high-risk patients undergoing balloon aortic valvuloplasty: results from the Global cVAD Registry. Int J Cardiol. 2017; 240: 120-125.

5 Balak W, Wiśniewska J, Ziółkowski M, et al. High-risk coronary angioplasty protected by an Impella pump combined with simultaneous iliac artery angioplasty. Kardiol Pol. 2019; 77: 726-727. 